\title{
Temperature evolution during simple shear test of steel
}

\author{
by S. P. Gadaj, W. K. Nowacki and E. A. Pieczyska
}

Center of Mechanics, Institute of Fundamental Technological Research, Swiętokrzyska 21, 00-049, Warsaw, Poland, e-mail: pgadaj@ippt.gov.pl

\begin{abstract}
Investigation of the simple plane shear of stainless steel and constructional steel was carried out. The stress-strain curves and the distributions of infrared radiation for various shear rates have been monitored. Temperature changes occurred in the sheared paths. This temperature raised due both to the increase of the deformation and to the rate of shear. The asymmetry of the thermal distribution of the shear paths related to the macroscopic shear bands was observed. Finally the results were compared with the results of numerical simulation of this kind of material deformed in adiabatic condition.
\end{abstract}

\section{Introduction}

Theoretical approaches concern the analysis of the shear testing under the isothermal or adiabatic conditions [1-3]. In reality, the heat emission and almost immediately its transmission to the surroundings accompany the process of shear. Thermomechanical coupling occurs there in nonadiabatic conditions. There are no published papers up to now on this problem, although such investigations were undertaken during dynamic tests [4].

In this paper, the investigations were performed of the static simple plane shear under nonadiabatic conditions. Their goal was to obtain the mechanical curves as well as the temperature distributions in the shear areas.

\section{Experimental details}

The tests were performed on sheet samples of two kinds of material: constructional steel XES and stainless steel $1 \mathrm{H} 18 \mathrm{~N} 9 \mathrm{~T}$ at four rates of deformation: $2.77 \times 10^{-3} \mathrm{~s}^{-1}, 5.55 \times 10^{-3} \mathrm{~s}^{-1}$, $11.1 \times 10^{-3} \mathrm{~s}^{-1}$ and $23.8 \times 10^{-3} \mathrm{~s}^{-1}$. There were two kinds of samples of XES steel: one of them (thickness $0.78 \mathrm{~mm}$ ) without any initial deformation and the second (thickness $0.58 \mathrm{~mm}$ ) initially deformed up to $\approx 25 \%$ by rolling. Samples of the 1 H118N9T steel were $0.5 \mathrm{~mm}$ thick. The samples were placed in a specially designed grip (transforming the compression into a simple plain shear) and fitted in the testing machine. Construction of this arrangement practically eliminates sliding of the specimen in the grip and ensures that the process of shear takes place on two parallel shear bands (paths) of the specimen, of $30 \mathrm{~mm}$ length and $3 \mathrm{~mm}$ width (Fig. 1). A change of temperature of the surface of these paths has been observed.

During the deformation we continuously monitored the load versus the displacement of the Instron crosshead, the load and this displacement versus time and the distribution of infrared radiation emitted by shear paths. The infrared radiation was measured using the thermovision camera AGA 680 coupled with a system of data acquisition and conversion PTR WIN (see [5]).

In order to secure higher and more homogeneous emissivity, the surface of the samples was blackened with carbon powder.

The distribution of intensity of the infrared radiation, recorded during investigation in digital form, allows us to reconstruct thermal pictures (thermograms) of the specimens.. In Fig. 2 an example of a thermogram of the shear zones of the specimen, deformed at the rate of $23.8 \times 10^{-3} \mathrm{~s}^{-1}$, and registered at the deformation $\gamma=0.84$ is shown. 
After measurement of each of the sheared paths a central line was drawn in the thermograms along the shear direction (Fig. 2). The temperature changes were found as an average value of the temperature along these lines.

The mean-square error of temperature evaluation, obtained this way, was $0.2-0.3 \mathrm{~K}$.

\section{Results and discussion}

The mechanical characteristics make it possible to derive the stress-strain relations. The stress-strain relations $\sigma_{12}(\gamma)$ found for all kinds of samples sheared at the same strain rate $11.1 \times 10^{-3} \mathrm{~s}^{-1}$ are shown in Fig. 3. The relations $\sigma_{12}(\gamma)$ obtained for the samples of XES steel, without any initial deformation (Fig. 3) and $1 \mathrm{H} 18 \mathrm{NgTsteel}$, show smooth parabolic transition from elastic to plastic deformation: materials of the samples hardens during all ranges of shearing. Another, initially deformed XES steel, shows rapidly increasing characteristics from the beginning and then an almost constant level.

Temperature changes of the shearing paths (Fig. 4) increase with both the increase of the deformation and the increase of the rate of the shear. The temperature increments obtained for the samples of XES steel after rolling are higher than for the samples without any initial deformation. In spite of the differences between the $\sigma_{12}(\gamma)$ characteristics, for both kinds of samples the character of the temperature increments are similar.

The temperature versus shear strain dependence found for $1 \mathrm{H} 18 \mathrm{~N} 9 \mathrm{~T}$ steel is different. For all values of shear rates the temperature increments for this steel are considerably higher. It is related to higher hardening rate of this steel (Fig. 3) and its lower heat conductivity; less amount of heat was carried to the grips.

The occurrence of the stress components $\sigma_{11}$ and $\sigma_{22}$ is caused by the condition $a=a_{0}=$ const. (the internal and external jaws of the grip move parallel, see Fig. 1). The existence of free edges of specimen $x_{1}= \pm l_{0} / 2$, where the normal stress component must vanish, induces the heterogeneity of the strain field. However, it is assumed that this boundary region is small compared to the length of the specimen. Theoretical considerations indicate that for $a_{0} / i_{0}=1 / 10\left(l_{0}=30 \mathrm{~mm}\right)$ this region covers almost $5 \%$ of the specimen length. An example of the strain field obtained numerically by the ABAQUS code for the steel $1 \mathrm{H} 18 \mathrm{~N} 9 \mathrm{~T}$ (under the assumption of elasto-plastic adiabatically deformed material at the strain level $\gamma=0.322$ ) is shown in Fig. 5a [6].

The disturbances of the stress and strain fields existing on free ends of the specimen during the shear test are visible in the temperature distribution. The temperature of these areas is higher than in the remaining parts of the specimen as seen in the initial stage of the process (Fig. 6). At higher deformations this effect is difficult to observe because of large temperature increments of the shear zones and due to the high thermal conductivity of this steel.

From theoretical considerations it follows that, during the simple shear test at deformations above $70-80 \%$, a localisation of the deformation develops ("macroscopic shear band") at a certain angle to the shear path. Fig. 5B presents a picture of this band for $\gamma=1.11$ shear deformation. The calculations were made for the elasto-plastic model of the body, under the assumption of combined isotropic-kinematic hardening and adiabatic process of deformation [6].

Suitable processing of thermal pictures recorded during the shear test indicates that the phenomenon of localised deformation is noticeable also in nonadiabatic conditions where the experiment has been conducted. For that purpose the following approach was adopted.

On the thermograms obtained in the non-homogeneous range of deformation $(\gamma \approx 1.50)$, 9 segments were chosen, intersecting the shear path perpendicular to the shear direction (Fig. 7; coloured). The co-ordinate $x_{2}$ of the beginning of each sector was the same (Figs. 1 and 7). Along these segments the distributions of temperature were determined in two approaches for each path, because the system PTR WIN allows to obtain such distributions simultaneously for 5 segments only. 
An example of the obtained thermograms with marked 5 segments and with the temperature distribution determined along them is shown in Fig. 7. The ends of the specimens are characterised by the inhomogeneous stress and strain state and moreover, the temperature distribution is there influenced by other factors than in the remaining area. That is why these ends were omitted in the analysis of temperature distribution.

The procedure described above of obtaining the temperature distribution was applied to both shear paths. Subsequently, these distributions were approximated by the product of the exponential function and the Gauss function. As the exact scaling of the distances were very difficult, the co-ordinates of the points in the picture and the distances between them were given in relative units.

In agreement with the results obtained theoretically, the macroscopic shear bands of both paths obtained for the same specimen were directed towards each other (Figs. 1 and $5 \mathrm{~b}$ ). Thus, the symmetrical superposition of the points indicating the temperature maxima in both paths and calculation of the mean value of the co-ordinate $x_{1}$ for these points (deviation of the temperature maximum from the shear direction) should eliminate a possible error caused by rotation of the paths relative to the shear direction.

The results of calculations obtained for extreme values of $\gamma$ where the macroscopic shear band occurs are shown in Fig. 8. These are the mean values for both paths. The coordinate system is the same as in Fig. 1; with $x_{1}$ along the shear direction and $x_{2}$ perpendicular to it. The position of maximum temperature found for the segment in the middle of the path was assumed as the origin of the co-ordinate system.

For each point, the statistical error of the determined position of maximum temperature was calculated (Fig. 8). Its value depends on the relative error of the temperature determination and decreases in accordance with a decrease in the temperature error. Other, nonstatistical errors of this determination were not taken into consideration.

The obtained values of the temperature maximum positions were approximated by the straight line. Results of this approximation were marked by solid lines, while the dashed line shows the position of the macroscopic shear band found from the theoretical calculations [6]. These results indicate that the line describing the position of the maximum temperature is not always located in the middle of the shear path.

Our results indicate that the slope of the macroscopic shear band changes with the deformation. Discrepancies between the theoretical and experimental investigations may be caused by the immediate flow of generated heat to the solid grips. At small temperature increments it can cause an effect of apparent change of inclination of the macroscopic shear band

\section{Conclusions}

For various kinds of steel at the same shear rate, a correlation between the mechanical characteristics and the temperature change is observed. For materials with higher strain hardening, higher temperatures of shear areas are observed i.e. more heat is emitted.

Investigations of temperature distribution on the surface of the shear paths confirm the existence of the theoretically predicted fields of strain heterogeneity. At the ends of shear zones these heterogeneities are manifested by the increase of temperature, particularly noticeable in the initial stage of shearing. As the deformation continues, the line describing the positions of maximum temperature deviates from the shear direction. It gives evidence for the development of the macroscopic shear band running along the specimen at a certain angle to the direction of shear. Differences between the angles predicted by the theory and those observed in the experiment are probably caused by the flow of heat to the solid grips, by the influence of temperature on the process of deformation and by the assumptions adopted in the theoretical model. Explanation of these divergences requires further investigations. 


\section{REFERENCES}

[1] HARREN (S.), LOVE (T. C.), et al - Analysis of Large-Strain Shear in Rate-Dependent Face-Centered Cubic Polycrystals: Correlation of Micro-and Macromechanics, Phil. Trans. R. Soc. Lond., A 328, 1989, p. 443.

[2] PAULUN (J. E.), PECHERSKI (R. B.) - On the relation for plastic spin, Arch. Appl. Mech. 62,1992, p. 376.

[3] NOWACKI (W. K.), NGUYEN HUU VIEM - Dynamic Simple Shear Test. Experiment and Numerical Inveatigation, Proc. 9th DYMAT Technical Conference, Munich, October 10-: 11 th 1995.

[4] HARTLEY (K. A.), DUFFY (J.), HAWLEY (R. H.) - Measurmend of the Temperature Profile during Shear Band Formation in Steel Deforming at High Strain Rates, J. Mech. Phys. Solids, 35, 3, 1987, p. 283.

[5] GADAJ (S. P.), NOWACKI (W. K.), PIECZYSKA (E.A.) - Changes of temperature during the simple shear test of stainless steel, Arch. Mech., 48, 4, 1996, p. 779-788.

[6] NGUYEN HUU VIEM, NOWACKI (W. K.) - Simple shear of metal sheets at higher rates of strain, Arch. Mech., 49, 2, 1997, p. 369-384.

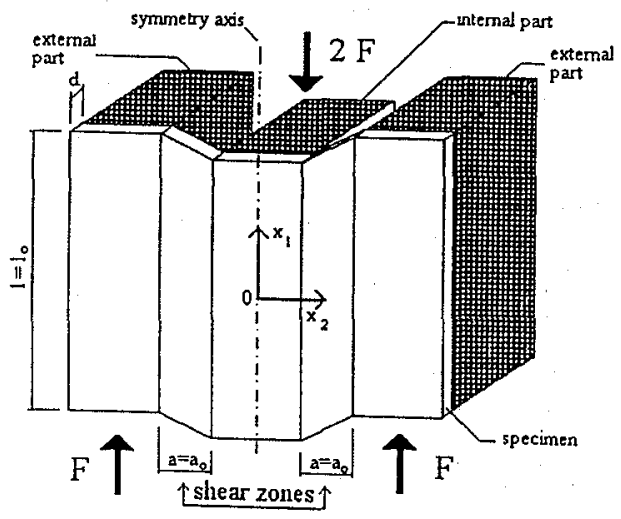

Fig. 1. Principle of shear test device

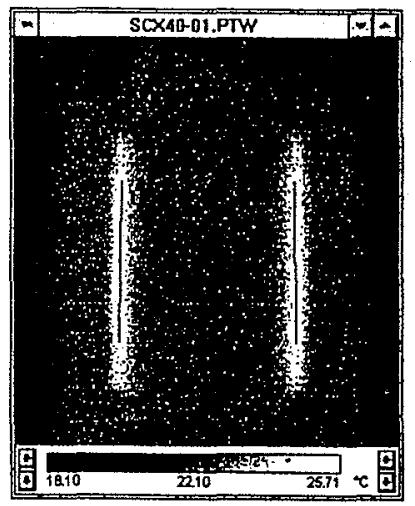

Fig. 2. A thermogram of sheared paths with central lines along them 
http://dx.doi.org/10.21611/qirt.1998.018

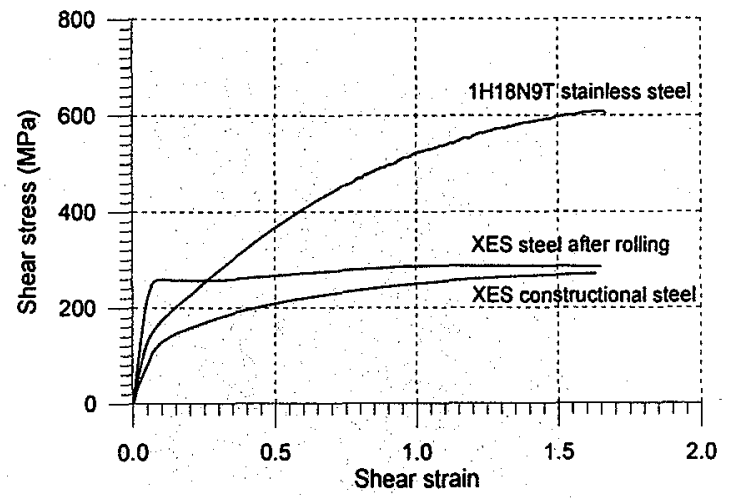

Fig. 3. Mechanical characteristics of investigated steels

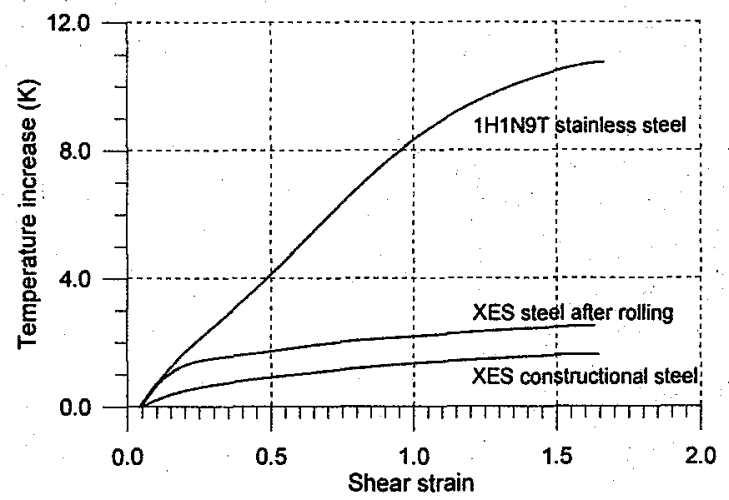

Fig. 4. Change in temperature of the sheared paths
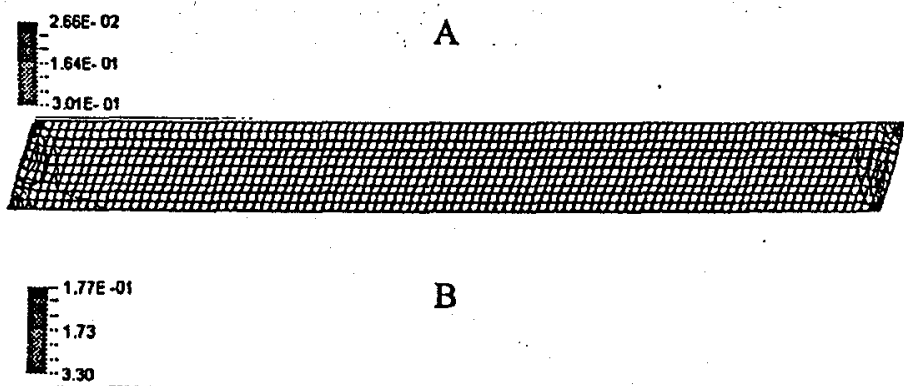

Fig. 5. Heterogeneity of deformation on free edges of sheared areas at $\gamma=0.322$ (A) and stress field in the shear areas at $\gamma=1.11$ (B); theoretical evaluation [6] 
http://dx.doi.org/10.21611/qirt.1998.018

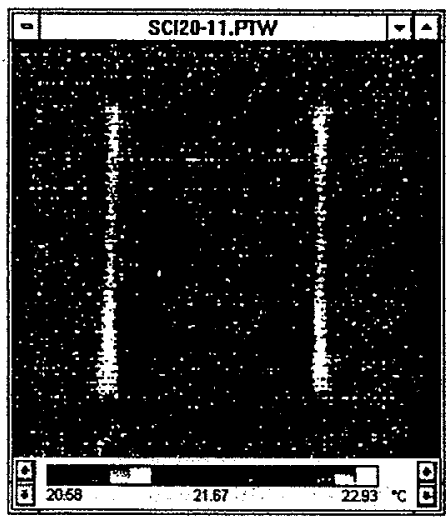

Fig. 6. A thermogram showing the zones of shear with higher temperature

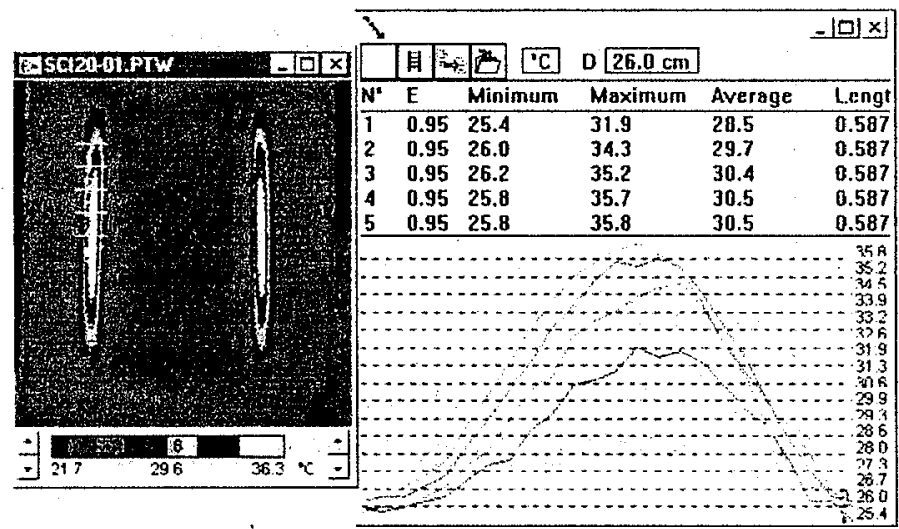

Fig. 7. Example of thermogram with 5 marked segments and temperature distribution determined along them

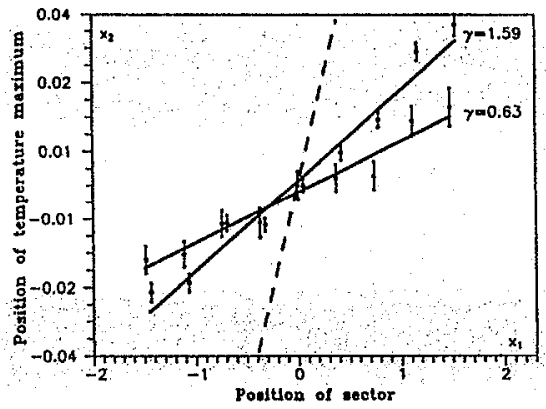

Fig. 8. Positions of maximum temperature in shear zones of the specimens at the strain rate $11.1 \cdot 10^{-3} \mathrm{~s}^{-1}$. The dashed line indicates the position of macroscopic shear band predicted by the theory 